\title{
Avaliação do perfil lípidico e renal de homens em treinamento de hipertrofia miofibrilar
}

\author{
Evaluation of the lipid and renal profile of men in \\ myofibrillary hypertrophy training
}

\author{
Kelly Carolina de Quadros Brolo ${ }^{1}$ \\ Sérgio Coitinho Junior ${ }^{2}$ \\ Rachel Gaudenzi ${ }^{3}$ \\ Jerri Luiz Ribeiro ${ }^{4}$ \\ Marcia Jacobsen ${ }^{5}$ \\ Valesca Veiga Cardoso ${ }^{6}$ \\ Marcello Mascarenhas ${ }^{7}$
}

\section{RESUMO}

Trata-se de um estudo transversal, que teve como objetivo de analisar possíveis alterações no colesterol total, HDL-c, triglicerídeos, ureia e creatinina, a partir de informações secundárias de um banco de dados de um projeto de pesquisa sobre os biomarcadores lipídicos e renais de homens em treinamento de hipertrofia miofibrilar de uma academia situada em Porto Alegre, que foram divididos em dois grupos: grupo teste que recebeu cápsulas contendo vitamina $\mathrm{C}$, vitamina $\mathrm{D}_{3}$, zinco e Serenoa repens, e grupo controle que recebeu cápsulas de amido de milho. Ambos os grupos administraram as cápsulas por via oral, de 12 em 12 horas. As dosagens bioquímicas foram realizadas no soro dos participantes durante dois momentos: antes dos participantes iniciarem a suplementação e após 45 dias de uso. As determinações bioquímicas seguiram as recomendações conforme o método enzimático colorimétrico da LABTEST $^{\circledR}$. Os resultados mostraram que pós a intervenção o grupo teste demonstrou um aumento significativo do colesterol total $(\mathrm{p}=0,048)$ e HDL-c $(0,055)$ e diminuição nos níveis de triglicerídeos $(\mathrm{p}=0,030)$ quando comparado ao controle. 0 grupo teste apresentou uma diminuição nas concentrações de creatinina $(p=0,527)$ e ureia $(p=0,044)$ quando comparado ao controle.

\section{PALAVRAS-CHAVE}

Colesterol, perfil renal, suplementos, vitaminas, Serenoa repens.

\footnotetext{
${ }^{1}$ Biomédica do Centro Universitário Metodista IPA - Porto Alegre, RS.

${ }^{2}$ Mestre pelo Programa de Pós Graduação em Biociências e Reabilitação do Centro Universitário Metodista IPA - Porto Alegre, RS. ${ }^{3}$ Biomédica e Bolsista CNCq pelo Programa de Pós Graduação em Biociências e Reabilitação do Centro Universitário Metodista IPA - Porto Alegre, RS.

${ }^{4}$ Educador Físico e Docente da Universidade Federal do Rio Grande do Sul - Porto Alegre, RS.

${ }^{5}$ Bióloga e Doutora e Pesquisadora da Universidade Federal do Rio Grande do Sul - Porto Alegre, RS.

${ }^{6}$ Biológa e Doutora e Pesquisadora da Universidade Federal do Rio Grande do Sul - Porto Alegre, RS.

${ }^{7}$ Farmacêutico Bioquímico, Docente do curso de Biomedicina e Programa de Pós-Graduação em Biociências e Reabilitação do Centro Universitário Metodista IPA - Porto Alegre, RS.
} 


\section{ABSTRACT}

This is a cross-sectional study aimed at analyzing possible changes in total cholesterol, HDL-c, triglycerides, urea and creatinine, based on secondary information from a database of a research project on lipid biomarkers and of men in myofibrillar hypertrophy training at a gym located in Porto Alegre, Brazil, who were divided into two groups: a test group that received capsules containing vitamin C, vitamin D3, zinc and Serenoa repens, and a control group that received capsules of corn starch. Both groups administered the capsules orally every 12 hours. Biochemical measurements were performed in the participants serum for two moments: before the participants started the supplementation and after 45 days of use. The biochemical determinations followed the recommendations according to the enzymatic colorimetric method of LABTEST $\circledast$. The results showed that after the intervention the test group demonstrated a significant increase in total cholesterol $(p=0.048)$ and HDL-c $(0.055)$ and decrease in triglyceride levels ( $p$ $=0.030)$ when compared to control. The test group showed a decrease in creatinine $(p=0.527)$ and urea $(p=0.044)$ concentrations when compared to control.

\section{KEYWORDS}

Cholesterol; Renal profile; Supplements; Vitamins; Serenoa repens. 


\section{INTRODUÇÃo}

0 treinamento resistido está designado a aumentar a massa muscular promovendo a síntese de proteínas do músculo miofibrilar ${ }^{1}$. Este processo hipertrófico é caracterizado a nível molecular pelo aumento da seção transversa do músculo esquelético e sua regulação é impulsionada por estímulos tensionais biomecânicos desenvolvidos pelo movimento do exercício e por aspectos hormonais e nutricionais ${ }^{2}$.

Além de ganho muscular, sabe-se que atividade física proporciona bem estar físico e mental, reduz as lipoproteínas de colesterol de baixa intensidade (LDL-c) e aumenta as lipoproteínas de colesterol de alta intensidade (HDL-c) além de manter peso corporal e a pressão arterial estáveis ${ }^{3}$. Estudos observacionais associaram fatores de riscos cardiovasculares, como dislipidemias, a baixas taxas de testosterona total, que é um hormônio androgênico, derivado do colesterol, responsável por estabelecer características sexuais masculinas como crescimento de pelos no rosto e desenvolvimento muscular, sua secreção decai gradualmente a partir dos 40 anos de idade no homem ${ }^{4,5}$.

0 uso de substâncias ergogênicas contribui para o bom desempenho dos praticantes de musculação. Assim estes recursos estão disponíveis como fitoterápicos, que é o produto obtido de planta medicinal, ou de seus derivados, exceto substâncias isoladas, com finalidade profilática, curativa ou paliativa, e a princípio, não prejudicam a saúde ${ }^{6-8}$. Umas destas é a Serenoa repens, que é uma planta pequena da família Arecaceae de nome científico Saw palmetto, e suas propriedades possuem efeitos antiandrogênicos, pró-apoptóticos e antinflamatório ${ }^{9,10}$.

A ingestão de micronutrientes é também, essencial para muitas funções fisiológicas e metabólicas do corpo, dentro deste grupo estão inclusas as vitaminas e os minerais ${ }^{11}$. A vitamina C, vitamina D3 e o zinco são importantes por atuarem nos processos antioxidantes, na manutenção do sistema imunológico, saúde óssea, contração muscular e neuromuscular ${ }^{12,13}$. Estes suplementos em geral, prometem grandes benefícios ao organismo, contudo o uso exagerado e sem o devido acompanhamento profissional pode ser nocivo à saúde, provocando aumento da síntese endógena de gordura. Estudos relataram que pode haver correlação entre o aumento de níveis séricos de vitamina D3 e o aumento dos parâmetros bio- químicos do perfil lipídico, e também pode ser prejudicial ao sistema renal, devido a seu metabolito ativo, o calcitriol, aumenta a absorção digestiva de cálcio, uma vez que a excreção urinária de cálcio está diretamente correlacionada com a absorção digestiva de cálcio, os metabólitos da vitamina $\mathrm{D}$ poderiam aumentar a calciúria e promover a formação de cálculos urinários ${ }^{14,15}$.

No contexto, o artigo objetivou avaliar as taxas de colesterol total, HDL-c, triglicerídeos, ureia e creatinina em homens submetidos ao uso concomitante de vitamina $\mathrm{C}$, vitamina $\mathrm{D}_{3}$, zinco e Serenoa repens que praticam treinamento de hipertrofia miofibrilar.

\section{MÉTODOS}

Trata-se de um estudo transversal, trabalhou com banco de dados de um projeto de pesquisa sobre os biomarcadores do perfil lipídico e renal de homens em treinamento de hipertrofia miofibrilar.

\section{Amostra}

Para a obtenção dos dados observados, a pesquisa realizada escolheu 20 indivíduos do sexo masculino, que foram selecionados após convite em mídia impressa e digital, com idade entre 25 e 55 anos, que eram praticantes do treinamento de hipertrofia miofibrilar de uma academia situada na cidade de Porto Alegre/ RS. E foram excluídos os sujeitos adultos com idade menor que 25 anos e maiores de 55 anos pessoas que estejam realizando terapia hormonal, uso de corticóides, benzodiazepinicos, antipsicóticos, antidepressivos, vitaminas lipossolúveis, vitaminas hidrossolúveis e minerais. Também foram excluídos participantes que estejam realizando outro tipo de treinamento ou sedentários também não se enquadrando no perfil proposto.

Após os participantes passaram por processo de randomização e cegamento dividindo-se em dois: grupo teste que recebeu cápsulas contendo 500 mg de vitamina $\mathrm{C}, 7,5 \mathrm{mg}$ de vitamina $\mathrm{D}_{3}, 7,5 \mathrm{mg}$ de zinco e $160 \mathrm{mg}$ de Serenoa repens, e grupo controle que recebeu cápsulas de amido de milho. Ambos os grupos administraram as cápsulas por via oral, de 12 em 12 horas. A manipulação destes produtos foi realizada em farmácia especializada de Porto Alegre/RS sob responsabilidade do farmacêutico. 


\section{Mensuração}

As dosagens foram realizadas no soro dos participantes durante dois momentos: antes dos participantes iniciarem a suplementação e após 45 dias de uso. As determinações bioquímicas seguiram as recomendações conforme o método enzimático colorimétrico e os biomarcadores analisados foram colesterol total, HDL-c, LDL-c, triglicerídeos, ureia e creatinina da marca LABTEST $^{\circledR}$. Todas as dosagens foram realizadas em triplicata e nas condições de qualidade laboratorial.

\section{Análise estatística}

Os dados foram analisados pelo teste de normalidade Kolmogorov-Smirnov, para analisar o tipo de distribuição, assim utilizou-se o teste t pareado para análise dos biomarcadores dos momentos pré e pós a intervenção de cada grupo e o teste tindependente para comparação entre os grupos, considerando o valor de $\mathrm{p}<0,05$. Os resultados foram expressos em média \pm desvio padrão.

\section{Aspectos éticos}

O estudo foi aprovado pelo Comitê de Ética, submetido à Plataforma Brasil sob número de parecer 1.818.630, CAAE sob número 60491316.6.0000.5308.

\section{RESULTADOS E DISCUSSÃo}

No presente estudo, os parâmetros bioquímicos do perfil lipídico e renal foram analisados do banco de dados em dois momentos: pré intervenção (Pré) nos grupos teste e controle e após 45 dias da intervenção (Pós) em ambos os grupos.

0 perfil lipídico (Tabela 1) demonstrou no momento pré uma diminuição das concentrações de colesterol total do grupo teste em relação ao controle $(p=0,045)$. 0 HDL-c no grupo teste estava menor do que o controle $(p=0,020)$ e os triglicerídeos apresentaram maior nível no grupo teste versus o controle $(\mathrm{p}=0,030)$.

.Tabela 1. Análise de Colesterol total, HDL-c e triglicerídeos do grupo controle e grupo teste nos momentos pré e pós a ingestão de vitamina C, vitamina D, zinco e Serenoa repens

\begin{tabular}{|l|l|l|l|r|}
\hline & \multicolumn{5}{|c|}{ Perfil lipídico (mg/dL) } \\
\hline & Grupo controle (média $\pm d p)$ & \multicolumn{2}{c|}{ Grupo teste (média $\pm d p)$} \\
\hline & Pré & Pós & Pré & $210,65 \pm 63,11$ \\
\hline Colesterol total (mg/dL) & $179,36 \pm 15,47$ & $205,66 \pm 43,57$ & $161,25 \pm 31,76$ & $60,69 \pm 6,09$ \\
\hline HDL-c (mg/dL) & $46,86 \pm 8,63$ & $55,16 \pm 5,75$ & $44,14 \pm 10,18$ & $150,81 \pm 30,11$ \\
\hline Triglicerídeos (mg/dL) & $102,72 \pm 13,58$ & $205,66 \pm 43,57$ & $115,12 \pm 24,42$ & \\
\hline
\end{tabular}

Fonte: Elaborado pela autoraCom base na Diretriz Brasileira de Dislipidemias e Prevenção de Aterosclerose de 2017, a média das concentrações do perfil lipídico de ambos os grupos apresentavam-se dentro dos valores de referência estabelecidos, demonstrando que os participantes não tinham nenhum risco de desenvolvimento de doenças ${ }^{16}$. Pós a intervenção o grupo teste demonstrou um aumento significativo do colesterol total em relação ao controle ( $p=0,048)$, o mesmo foi identificado no HDL-c $(\mathrm{p}=0,055)$ e os níveis de triglicerídeos foram menores $(\mathrm{p}=0,030)$ versus controle.

Para as analises de LDL-c, os resultados não foram considerados para este estudo, pois foi utilizado o cálculo a partir da fórmula de Friedewald, que apresenta limitações e sofre interferências dos triglicerídeos, assim os resultados não foram confiáveis. ${ }^{16}$

Através da comparação dos momentos final em relação inicial para o grupo controle apresentou um aumento do colesterol total, HDL-c e triglicerídeos, o mes- mo aconteceu no grupo teste, porém não foram significativos. Os parâmetros bioquímicos do perfil lipídico são determinantes para averiguar anormalidades nos níveis sanguíneos de colesterol e triglicerídeos, quando há alterações significativas pode-se ter uma estimativa de riscos de doenças como a aterosclerose ${ }^{17}$.

Segundo o estudo de Kwon e Lee ${ }^{3}$, os lipídeos são afetados pelo exercício físico, porém o colesterol total 
não é facilmente reduzido pelo treinamento, nem os exercícios intensos não melhoram significativamente sua concentração sérica. No presente estudo houve um aumento significativo nas concentrações de colesterol total para ambos os grupos. Comparando com o estudo de Kwon e Lee ${ }^{3}$, possivelmente o exercício não altera beneficamente as concentrações de colesterol total. Como os dois grupos obtiveram aumento nas concentrações, não há distinção se o uso de vitamina C, vitamina D, zinco e Serenoa repens ou placebo podem influenciar nesta elevação, uma vez que os hábitos alimentares também influenciam no colesterol.

Quanto ao HDL-c, os grupos tiveram um aumento significativo na concentração sérica, mostrando que o exercício influenciou nesta alteração, que segundo Jan et. $\mathrm{al}^{18}$ outros estudos confirmaram este efeito positivo do exercício físico ao HDL-c, diminuindo o risco de doenças cardiovasculares nos indivíduos. Para Ahn e Kim ${ }^{19}$ o aumento do HDL-c está relacionado à saúde, mostrando que em atletas há um aumento maior do que em indivíduos comuns. Este aumento é atribuído à ativação de enzimas hidrolíticas de lipoproteínas no plasma, convertendo componentes de quilomícrons e lipoproteínas de baixa densidade em HDL-C, além da supressão da atividade de triglicerídeos hepática, a qual pode resultar em baixo catabolismo de HDL-C ${ }^{3}$. Segundo o estudo de $\mathrm{McRae}^{20}$ a ingestão de vitamina $\mathrm{C}$ colabora para um aumento dos níveis de HDL-c. Esta vitamina é considerada um potente antioxidante, que contribuem para a redução dos efeitos do estresse e da falta de oxigênio provocadas pelo exercício intenso e continuo que causam danos musculares ${ }^{21}$.
Com relação aos triglicerídeos, segundo Wange e $\mathrm{Xu}^{22}$ o exercício pode induzir a redução sua concentração sérica, principalmente em exercícios que exigem grande quantidade de energia haverá maior diminuição dos triglicerídeos. Para Saeidlou et al. ${ }^{15}$ alguns estudos transversais demonstraram que há uma associação entre um aumento no nível de vitamina D e um declínio nos triglicerídeos. 0 possível mecanismo desse efeito é que esta vitamina pode aumentar o cálcio sérico, aumentando a absorção intestinal de cálcio, reduzindo a formação e a secreção hepática de triglicerídeos, o que poderia então reduzir os triglicerídeos séricos. Porém outros experimentos de intervenção não esclareceram a relação entre lipídeos e suplementação com vitamina D para melhorar o perfil lipídico. Comparando com estudos já realizados, os resultados encontrados neste estudo demonstraram um aumento significativo na concentração sérica dos triglicerídeos dos grupos. No grupo controle esta elevação foi maior que no grupo teste, demonstrando que possivelmente a associação das vitaminas, Serenoa repens e exercício físico podem ter colaborado para que não houvesse um aumento maior como no grupo controle.

Quanto ao perfil renal, Tabela 2, a etapa pré constatou-se que as concentrações de creatinina apresentavam uma diminuição no grupo teste quando comparadas ao controle $(p=0,615)$. A ureia estava menor do que o grupo controle $(\mathrm{p}=0,40)$. Quanto ao momento pós a intervenção o grupo teste apresentou uma diminuição nas concentrações de creatinina quando comparado ao controle $(\mathrm{p}=0,527)$, e os níveis de creatinina foram menores $(p=0,044)$ em relação ao controle.

Tabela 2. Análise de creatinina e ureia do grupo controle e grupo teste nos momentos pré e pós a ingestão de vitamina C, vitamina D, zinco e Serenoa repens.

\begin{tabular}{|c|c|c|c|c|}
\hline \multicolumn{5}{|c|}{ Perfil renal (mg/dL) } \\
\hline Parâmetro & \multicolumn{2}{|c|}{ Grupo controle (média/dp) } & \multicolumn{2}{c|}{ Grupo teste (média/dp) } \\
\hline & Pré & Pós & Pré \\
\hline Creatinina (mg/dL) & $0,93 \pm 0,25$ & $1,1 \pm 0,36$ & $0,85 \pm 0,22$ & $0,99 \pm 0,33$ \\
\hline Ureia (mg/dL) & $43,34 \pm 11,94$ & $64,22 \pm 9,44$ & $49,09 \pm 10,68$ & $55,46 \pm 14,80$ \\
\hline
\end{tabular}

Fonte: Elaborado pela autora 
Em relação à comparação entre os momentos estudados foi possível observar que o grupo controle apresentou aumento na concentração da creatinina e ureia, sendo somente a ureia estatisticamente significativa e o grupo teste apresentou elevação nos dois analitos, mas não ocorreram diferenças.

A creatinina e a uréia avaliada no soro têm importante significado para o funcionamento dos rins. Quando os níveis estão elevados na corrente sanguínea demonstram que há uma disfunção renal ${ }^{23}$.

Quanto à creatinina, a concentração pré dos grupos encontrava-se dentro dos valores de referência, demonstrando bom funcionamento renal. No pós os grupos demonstraram um aumento nas concentrações, mesmo assim o resultado não foi estatisticamente significativo, ainda permanecendo dentro dos valores de referência. Este aumento pode estar relacionado ao exercício físico praticado ${ }^{24}$. 0 uso de vitamina D também pode aumentar o nível sérico de creatinina, e segundo Letavernier e Daudo ${ }^{14}$ alguns estudos randomizados controlados relataram um aumento do risco de cálculos renais como um evento adverso resultante da administração desta vitamina. Para Hesswaniet et al. ${ }^{25}$ indivíduos com histórico de cálculo renal devem ter cuidado para a administração de suplementação desta vitamina, pois correm o risco de formação de novos cálculos renais. Em comparação com estes estudos, deve-se ter um cuidado com o uso de vitamina D, pois no grupo teste houve uma elevação maior do que no grupo controle, visto que a pesquisa teve duração de 45 dias e o uso prolongando pode causar dano renal.

$\mathrm{O}$ uso de vitamina $\mathrm{C}$ também é sugestivo de dano renal, segundo Ferraro et. $\mathrm{al}^{26} \mathrm{o}$ uso prolongado pode atuar como um fator de risco para a formação de cálculos renais, porque é capaz de aumentar a excreção urinária de oxalato. Porém, segundo Jaturakan et. $\mathrm{al}^{13}$, um estudo com ratos demonstrou que o uso deste suplemento instigou o comprometimento glomerular e tubular, o estado oxidativo e as alterações histológicas do túbulo, e que seu uso seria mais eficaz em combinação com a vitamina E, pois neste mesmo estudo a associação destas vitaminas mostrou que todas as disfunções foram recuperadas. Contudo, sabe-se que a Vitamina C é um poderoso antioxidante e limpador de radicais livres que protege nossos tecidos, membranas celulares e DNA de danos oxidativos ${ }^{27}$.
Quanto à uréia, no momento pré, a concentração média dos grupos apresentava um leve aumento, estando acima dos valores de referência permitidos. No momento pós os grupos apresentaram valores ainda maiores, apresentando resultados estatisticamente significativos. Segundo Giardino et. $\mathrm{al}^{28}$, quando a função renal está comprometida, concentrações crescentes de uréia no sangue se acumulam constantemente. Vários estudos nos últimos dez anos mostraram que a uréia pode participar da progressão do processo aterosclerótico. Além disso, níveis elevados de uréia podem induzir à resistência à insulina e comprometer a secreção pancreática de insulina, o que poderia contribuir para a alta morbidade cardiovascular. Conforme este estudo, houve uma elevação da concentração sérica de uréia nos grupos em curto prazo, porém no grupo teste está elevação foi menor versus o grupo controle.

O uso de Serenoa repens poderia ser um dos critérios para diferenciar a elevação da ureia no grupo teste, visto que esta planta é utilizada no tratamento da hiperplasia benigna da próstata e sintomas urinários, como fluxo urinário fraco, hesitação e noctúria. Geralmente seu uso é bem tolerado e não foi associado a nenhum evento adverso grave $27,29,30,31,32$. Segundo Avins et. al $^{29}$ é improvável que haja uma toxicidade da utilização por um período de até 18 meses, e não houve diferenças significativas na dosagem de creatinina entre grupos experimentais que utilizaram Serenoa repens ou placebo.

O Zinco poderia ser outro fator importante que poderia ter ajudado para que o grupo teste tivesse uma elevação menor ureia em relação ao grupo controle. É um micronutriente essencial para o crescimento e desenvolvimento normal, bem como para alcançar e manter a saúde $^{30,33,34}$. Segundo Prasad ${ }^{35}$ pacientes com insuficiência renal apresentavam concentração reduzida de zinco no plasma. Segundo Rao et. $\mathrm{al}^{36}$ produz efeito protetor aos rins, porém é conflitante e dependente da quantidade. Doses baixas pode não atingir o limiar terapêutico e doses mais elevadas podem tornar-se ineficaz devido à toxicidade, portanto seu uso deve ser cauteloso.

\section{CONCLUSÃo}

Estudos mostraram que exercício físico é benéfico para aumento de HDL-c e redução dos triglicerídeos, porém no presente estudo não ocorreu essa modifica- 
ção. e que os hábitos alimentares, o consumo de álcool e tabaco não foi controlado e nem foi critério de exclusão do estudo. Apesar das vitaminas serem essenciais para o organismo, alguns estudos evidenciaram que doses inadequadas poderiam ser prejudiciais ao sistema renal, e o seu uso deve ser cauteloso, principalmente quando utilizadas em conjunto
Assim o uso de vitamina D, vitamina C, zinco e $\mathrm{Se}$ renoa repens em curto prazo, associado ao exercício físico de hipertrofia miofibrilar é conflitante. Em virtude que não ocorreu alterações significativas, o uso simultâneo destes suplementos não foi benéfico para organismo 


\section{REFERÊNCIAS}

1. Damas F, Phillips SM, Libardi CA, et al. Resistance training-induced changes in integrated myofibrillar protein synthesis are related to hypertrophy only after attenuation of muscle damage. J Physiol [internet] 2016 jul [acesso em 2018 abr 12]; 594(18):5209-22. Disponível em: https://www.ncbi.nlm.nih. gov/pmc/articles/PMC5023708/pdf/TJP-594-5209.pdf.

2. Paes, S. T. Efeitos do consumo proteico sobre a hipertrofia ocasionada pelo treinamento resistido: uma visão atual. RBNE-Revista Brasileira de Nutrição Esportiva. [internet] 2016 jan [acesso em 2018 abr 27]; 10(55), 11-23. Disponível em: http://www.rbne.com.br/index.php/rbne/article/ view/595.

3. Kwon H, Lee H. Effect of vigorous physical activity on blood lipid and glucose. [internet] $2017 \mathrm{dez}$ [acesso em $2018 \mathrm{mar}$ 11];13(6):653-8. Disponível em: https://www.e-jer.org/upload/jer-13-6-653.pdf.

4. Duan CW, Xu L. Testosterone and androstanediol glucuronide among men in NHANES III. BMC Public Health. [internet] 2018 mar [acesso em 2018 abr 15]; 18(1):1-6. Disponível em: https://bmcpublichealth.biomedcentral.com/track/ pdf/10.1186/s12889-018-5255-6.

5. AIRES, Margarida de Mello. Fisiologia. 4 ed. Rio de Janeiro,

6. Brasil, Agência Nacional de Vigilância Sanitária. Formulário de Fitoterápicos da Farmacopeia Brasileira. [internet] 2011 [acesso em 2018 abr 17]; 1:126. Disponível em: http://www. anvisa.gov.br/hotsite/farmacopeiabrasileira/conteudo/Formulario_de_Fitoterapicos_da_Farmacopeia_Brasileira.pdf.

7. Conde, B. E., Macedo, A. L., Fonseca, A. S. et. al. Estudo crítico sobre utilização de fitoterápicos por praticantes de exercício físico em academias de musculação. Biológicas \& Saúde [internet] 2015 [acesso em 2018 abr 1]; 5(16). Disponível em: http://seer.perspectivasonline.com.br/index.php/biologicas_e_ saude/article/view/562.

8. Pereira, M. A. O., Pereira, É. A. A., Moreira et. al. Influência do uso de lepidum meyenii walp e tribulus terrestris em praticantes de musculação. RBNE-Revista Brasileira de Nutrição Esportiva. [internet] 2017 jan [acesso em 2018 abr 01]; 11(67), 836842. Disponível em: http://www.rbne.com.br/index.php/rbne/article/view/910.

9. Rodríguez Leyes, E. A., González Canavaciolo, V. L., Marrero Delange, D., et. al. Fracciones lipídicas obtenidas a partir de frutos de Serenoa repens recolectados en Cuba. Revista Cubana de Plantas Medicinales. [internet] 2012 mar [acesso $2018 \mathrm{em}$ abr 12]; 17(1), 11-20. Disponível em: http://scielo.sld.cu/scielo.php?script=sci_arttext\&pid=S1028-47962012000100002.

10. Booker, A., Suter, A., Krnjic, A. et. al. Comparação fitoquími-

ca de produtos de Saw Palmetto utilizando cromatografia gasosa e perfil metabólico de espectroscopia de ressonância magnética nuclear de 1H. Journal of Pharmacy and Pharmacology. [internet] 2014 Jun [acesso em 2018 març 05]; 66 (6), 811-822. Disponível em: https://www.ncbi.nlm.nih.gov/pubmed/24417505.

11. Ciudad R. A. Infecciones en ginecología y obstetricia: producción científica de la Sociedad Peruana de Obstetricia y Ginecología en sus setenta años de vida institucional. Rev. peru. ginecol. obstet. [Internet]. 2017 Jul [acesso em 2018 Jun 03]; 63(3): 449-454. Disponivel em: http://www.scielo.org.pe/ scielo.php?script=sci_arttext\&pid=S2304-

12. Souza, C. A., Siqueira, S., de Amorim, A. F., Morais, et. al. Encapsulation of l-ascorbic acid within the natural biopolymer-galactomannan-using the spray-drying method: preparation, characterization, and evaluation of antioxidant activity. Química Nova.[internet] 2015 ago [acesso em 2018 abr 17] 38(7), 877-883. Disponível em: http://www.scielo.br/ scielo.php?pid=S0100-40422015000700877\&script=sci_arttext.

13. Jaturakan $O$, Dissayabutra $T$, Chaiyabutr $N$, Kijtawornrat $A$, et al. Combination of vitamin $\mathrm{E}$ and vitamin $\mathrm{C}$ alleviates renal function in hyperoxaluric rats via antioxidant activity. J Vet Med Sci. [internet] 2017 mai [acesso 2018 abr 08]; 79(5):896-903. Disponível em: https://www.jstage.jst.go.jp/article/ jvms/79/5/79_17-0083/_pdf/-char/em.

14. Letavernier E, Daudon M. Vitamin d, hypercalciuria and kidney stones. Nutrients. [internet] 2018 mar [acesso em 2018 abr 08]; 10 (3):1-11. Disponível em: http://www.mdpi. com/2072-6643/10/3/366.

15. Nouri Saeidlou S, Vahabzadeh D, Babaei F, et. al. Seasonal variations of vitamin $D$ and its relation to lipid profile in Iranian children and adults. J Health Popul Nutr. [internet] 2017 mai [acesso em 2018 abr 17];36(1):21. Disponível em: https:// jhpn.biomedcentral.com/track/pdf/10.1186/s41043-0170096-y.

16. SBC. Atualização da diretriz brasileira de dislipidemias e erevenção da aterosclerose. Arq Bras Cardiol.[internet] 2017 ago [acesso em 2018 mai 29]; 109(1):76. Disponível em: http://publicacoes.cardiol.br/2014/diretrizes/2017/02_DIRETRIZ_DE_DISLIPIDEMIAS.pdf.

17. Huff T, Jialal I. Fisiologia, Colesterol. Em: StatPearls. Ilha do Tesouro (FL): Publicação StatPearls; [Internet] 2017 nov [acesso em 2018 fev 25]. Disponível em: https://www.ncbi.nlm.nih. gov/books/NBK470561/

18. Jan C-F, Chang H-C, Tantoh DM, et al. Duration-response association between exercise and HDL in both male and female Taiwanese adults aged 40 years and above. Oncotarget. [internet] $2018 \mathrm{dez}$ [acesso em 2018 mar 11]; 9(2):2120-7. Disponível em: http://www.oncotarget.com/index.php?journal= 
oncotarget $\&$ page $=$ article $\&$ op $=$ view $\&$ path []$=23251 \&$ pa th[]=73296.

19. Ahn N, Kim K. High-density lipoprotein cholesterol (HDL-C) in cardiovascular disease: effect of exercise training. Integr Med Res. [internet] 2016 Jul [acesso em 2018 abr 22]; 5(3):212-5. Disponível em: https://www.sciencedirect.com/ science/article/pii/S2213422016300737?via\%3Dihub.

20. McRae MP. Vitamin C supplementation lowers serum low-density lipoprotein cholesterol and triglycerides: a meta-analysis of 13 randomized controlled trials. J Chiropr Med. [internet] 2008 mai [acesso em 2018 abr 08]; 7(2):48-58. Disponível em: https://www.journalchiromed.com/article/ S0899-3467(08)00028-1/fulltext.

21. Quadros, L., \& de Barros, R. L. S. Vitamina C e performance: uma revisão. RBNE-Revista Brasileira de Nutrição Esportiva. [internet] 2016 jan [acesso em 2018 mai 01], 10(55), 112-119. Disponível em: http://www.rbne.com.br/index.php/rbne/article/view/557.

22. Wang Y, Xu D. Effects of aerobic exercise on lipids and lipoproteins. Lipids Health Dis. [internet] 2017 [acesso em 2018 abr 22];16(1):1-8. Disponível em: https://lipidworld.biomedcentral.com/articles/10.1186/s12944-017-0515-5.

23. Duong, H. D., \& Rhee, J. I. Development of Ratiometric Fluorescent Biosensors for the Determination of Creatine and Creatinine in Urine. Sensors. [internet] 2017 nov [acesso em 2018 mai 13]; 17(11), 2570. Disponível em: https://lipidworld.biomedcentral.com/articles/10.1186/s12944-017-0515-5.

24. Lee, E. C., Fragala, M. S., Kavouras, et. al. Biomarkers in Sports and Exercise: Tracking Health, Performance, and Recovery in Athletes. Journal of strength and conditioning research. [internet] 2017 jul [acesso em 2018 abr 02]; 31(10), 2920. Disponível em: https://journals.lww.com/nsca-.

25. Hesswani C, Noureldin YA, Elkoushy MA, et. al. Combined vitamin $\mathrm{D}$ and calcium supplementation in vitamin $\mathrm{D}$ inadequate patients with urolithiasis: Impact on hypercalciuria and de novo stone formation. J Can Urol Assoc. [internet] $2015 \mathrm{dez}$ [acesso em 2018 abr 04]; 9(11-12December):403-8. Disponível em: http://cuaj.ca/index.php/journal/article/ view/3332/2462.

26. Ferraro PM, Curhan GC, Gambaro G, et. al. HHS Public Access. [internet] 2017 out [acesso em 2018 abr 08]; 67(3):4007. Disponível em: http://www.ncbi.nlm.nih.gov/pmc/articles/ PMC4769668/.

27. Souyoul SA, Saussy KP, Lupo MP. Nutraceuticals: A Review. Dermatol Ther (Heidelb). [internet] $2018 \mathrm{fev}$ [acesso em 2018 mai 13]; 8(1):5-16. Disponível em: https://link.springer.com/ article/10.1007\%2Fs13555-018-0221-x.
28. Giardino I, D’Apolito M, Brownlee M, et al. Vascular toxicity of urea, a new "old player" in the pathogenesis of chronic renal failure induced cardiovascular diseases. Turk Pediatr Ars. [internet] 2017 dez [acesso em 2018 mai 13]; 52(4):187-93. Disponível em: http://www.turkpediatriarsivi.com/eng/makale/4024/309/Full-Text.

29. Avins AL, Lee JY, Meyers CM, et. al. Safety and toxicity of saw palmetto in the CAMUS trial. J Urol. [internet] 2012 out [acesso em 2018 mai 06]; 189(4):1415-20. Disponível em: https:// www.jurology.com/article/S0022-5347(12)05181-6/fulltext.

30. Tran CD, Gopalsamy GL, Mortimer EK, et. al. The potential for zinc stable isotope techniques and modelling to determine optimal zinc supplementation. Nutrients. [internet] $2015 \mathrm{mai}$ [acesso em 2018 abr 09]; 7(6):4271-95. Disponível em: http:// www.mdpi.com/2072-6643/7/6/4271.

31. Krzysztofik M, Wilk M, Wojdała G, Gołaś A. Maximizing Muscle Hypertrophy: A Systematic Review of Advanced Resistance Training Techniques and Methods. Int J Environ Res Public Health. 2019 Dec 4;16(24):4897. [acesso em 2021 jun 19]. Disponível em: https://pubmed.ncbi.nlm.nih.gov/31817252

32. Wackerhage H, Schoenfeld BJ, Hamilton DL, Lehti M, Hulmi JJ. Stimuli and sensors that initiate skeletal muscle hypertrophy following resistance exercise. J Appl Physiol (1985). 2019 Jan 1;126(1):30-43. [acesso em 2021 jul 29]. Disponível em: https://pubmed.ncbi.nlm.nih.gov/30335577.

33. Liao CD, Chen HC, Kuo YC, Tsauo JY, Huang SW, Liou TH. Effects of Muscle Strength Training on Muscle Mass Gain and Hypertrophy in Older Adults With Osteoarthritis: A Systematic Review and Meta-Analysis. Arthritis Care Res (Hoboken). 2020 Dec;72(12):1703-1718. [acesso em 2021 jul 30]. Disponível em: https://pubmed.ncbi.nlm.nih.gov/31628720.

34. Baz-Valle E, Fontes-Villalba M, Santos-Concejero J. Total Number of Sets as a Training Volume Quantification Method for Muscle Hypertrophy: A Systematic Review.J Strength Cond Res. 2021 Mar 1;35(3):870-878. [acesso em 2021 jul 31]. Disponível em: https://pubmed.ncbi.nlm.nih.gov/30063555.

35. Prasad AS. Discovery of Human Zinc Deficiency: Its Impact on Human Health and Disease. Adv Nutr. [internet] 2013 mar [acesso em 2018 abr 08]; 176-90. Disponível em: https://academic.oup.com/advances/article/4/2/176/4591626.

36. Rao K, Sethi K, Ischia J, et al. Protective effect of zinc preconditioning against renal ischemia reperfusion injury is dose dependent. PLoS One. [internet] 2017 jul [acesso em 2018 mai 13]; 12(7):1-15. Disponível em: http://journals.plos.org/plosone/article?id=10.1371/journal.pone. 0180028 . 